\title{
A Modelica Library of Anisotropic Flexible Beam Structures for the Simulation of Composite Rotor Blades
}

\author{
Christian Spieß Manfred Hajek \\ Technische Universität München, Institute for Helicopter Technology \\ Boltzmannstr. 15, 85748 Garching
}

\begin{abstract}
Beam theories are extensively used for simulation of helicopter rotor blades. The predominant deployment of composite materials in rotor blade development demands for complex theories that are able to describe the elastic behavior of anisotropic and nonhomogeneous materials. In this paper a Modelica library is presented which is capable of simulating extensional, torsional and flexural deformation and the couplings between those degrees of freedom. The structural dynamic model is based on cross-sectional analysis.

Keywords: flexible structures; rotating beams; helicopter rotor design; cross-sectional analysis
\end{abstract}

\section{Introduction}

The non-linear static and dynamic analysis of bent and twisted beams is of major importance for many engineering disciplines. Especially for helicopter rotor applications beam models are used to simulate its dynamic behavior. Since helicopter rotor blades are made of composite structures and materials that may be anisotropic or nonhomogeneous the long and slender beam structure is subject to non-classical effects such as transverse shear deformation, geometric nonlinearities, cross-sectional warping, and elastic coupling [4]. Thus classical beam theories limited to isotropic materials and simple cross-sectional geometries may not be applicable in rotorcraft analysis codes. Some vibration phenomena in particular with significant bending-torsion coupling require adequate modeling. Therefore a sophisticated beam theory has been implemented to Modelica which has extensively been tested in practical applications such as the CAMRAD II [7] rotorcraft analysis code and been proven to provide satisfactorily results [8]. One of the key features of modern beam theories is the cross-sectional analysis. It splits the problem into a two-dimensional analysis of the cross-section and the one-dimensional beam kinematics. The two-dimensional analysis provides the structural and inertial parameters that can be set at any number of points on the beam. Hence the influence of anisotropy and inhomogeneity can be taken into account and these methods are not limited to specific materials. The one-dimensional analysis provides the elastic equations of motion to calculate the kinematics of the beam. The main advantage of this approach as opposed to three-dimensional finite element analysis is the significant reduction of computational effort. It allows the calculation of elastic beam behavior in multi-body environments as well as real time applications.

\section{Modeling Capabilities}

The flexible beam library is a Modelica package to model elastic motion of beam-like structures represented by axial, bending, and torsion deflection of a beam with arbitrary cross-sectional geometry. To attach the beam model to the simulation environment the standard Modelica frame connector from the MultiBody library is used. In addition to the connectors at each end of the beam an arbitrary number of frames on the beam axis may be defined to connect to other system components such as joints, sensors, or force elements. Figure 1 shows an exemplary setup with two beam segments connected in series. The user has the ability to define an arbitrary number of cross-sectional properties along the beam axis including the stiffness parameters and inertial properties. Those parameters can be obtained by NABSA [5] or VABS [1], which are both 2D cross-sectional analysis tools for general nonhomogeneous and anisotropic beam sections including warp and twist.

The library features two options to model the beam:

1. An Euler-Bernoulli beam theory for isotropic beam materials with St. Venant torsion. 


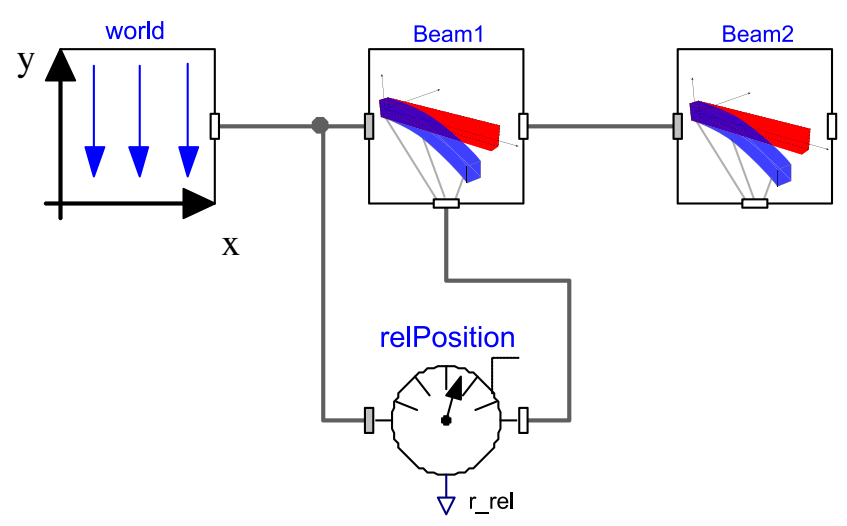

Figure 1: example beam setup

2. A beam theory for anisotropic or composite materials including transverse shear deformation.

The effects of cross-section warping and transverse shear are included in the section structural properties. Their effect on inertial forces and interface geometry is neglected, warp variables are expressed in terms of strain measures. The undeflected beam axis is assumed to be straight within the component. Thus a beam geometry defined by initial curvature or kinks must be split into several straight beam components. Also the theory requires the integration of beam properties along the beam axis which is implemented as Gaussian integration. Hence structural and inertial properties must not vary rapidly along its length.

\section{Beam Theory}

The motion of the beam element is represented by the rigid body motion of a reference frame at one end of the beam and an elastic motion relative to this frame. Due to this decomposition it is possible to superimpose an arbitrary large motion of the reference frame, which will be treated in a correct manner, by a small elastic motion approximated to the second order. The representation of the elastic deformation is based on references $[7,6]$. The reference frame defines the coordinate system $B$ at the origin of the undeflected beam axis. This axis extends on the positive $x$-axis of system $B$ from $x=0$ to $x=l$. The elastic motion of a point on the beam axis is described by four parameters; namely the constant position $x$ on the undeflected axis plus the elastic elongation $u$ as well as the bending deflections $v$ and $w$ that cause a rotation of the crosssection. Additionally the parameter $\theta$ is used to describe the rotation around the beam axis (see Fig. 2). The tangent of the cross-section at this point is rotated by the angles $\beta$ and $\zeta$ around the $y$ and $z$ axes respec-

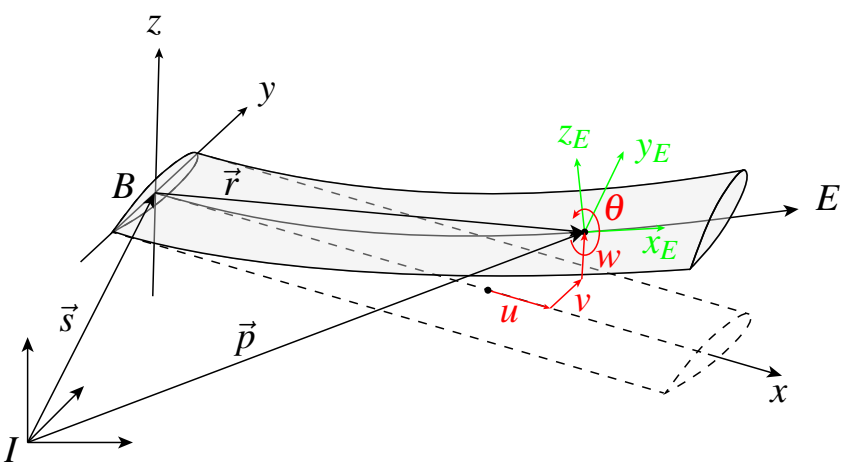

Figure 2: motion of the beam

tively produced by bending deflection, which can be obtained by $\sin \beta=w^{\prime}$ and $\sin \zeta=v^{\prime}$ for second order approximation ${ }^{1}$. That leads to the transformation matrix $C^{E B}(x)$ between the origin of the beam and the bent and twisted axes of the coordinate system $E$ at position $x$ of the beam axis. Euler angles are used to describe the rotation $C^{E B}=X_{\theta} Y_{-\beta} Z_{\zeta}$, where $X, Y$, and $Z$ are the rotation matrices around the indexed angles respectively.

\subsection{Cross-Section Motion}

To describe the motion of an arbitrary point on the cross section the previous definition needs to be extended by the effects of transverse shear and warping. Thus the vector $r$ from the origin of the beam relative to a point on the cross-section is constructed by:

a) the constant axial position $x$ and the elongation $u=u_{e}+U$, where $u_{e}$ is due to elastic elongation and $U$ is the elongation due to bending deflections

b) the transverse shear deformation rotating the cross-section with $v$ around the $z$-axis and with $\omega$ around the $y$-axis

c) the elastic transversal deformation $v$ and $w$

d) the rotation $\theta=\phi+\theta_{C / I}+\Theta$ around the $x$-axis, where $\phi$ is the elastic torsion, $\theta_{C}$ and $\theta_{I}$ is the pitch of the structural and inertial principal axes respectively, and $\Theta$ is the rotation due to bending deflections

e) the position of the point on the rotated crosssection represented by the coordinates $\eta$ and $\zeta$ and the warping displacements $W$

\footnotetext{
${ }^{1}$ the notation $(\cdot)^{\prime}$ is used for the derivative with respect to $x$
} 


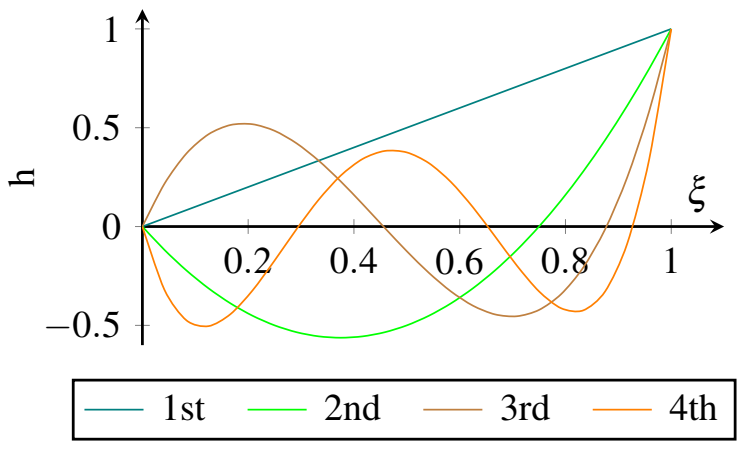

Figure 3: shape functions for $u_{e}$ and $\phi$

So the vector $r$ with respect to the coordinate system $B$ can be written as:

$$
r=\left(\begin{array}{c}
x+u \\
v \\
w
\end{array}\right)+C\left(\begin{array}{l}
0 \\
\eta \\
\zeta
\end{array}\right)+C\left(\begin{array}{l}
W_{1} \\
W_{2} \\
W_{3}
\end{array}\right)
$$

with

$$
C=\left(Z_{-v} Y_{\omega}\right) C^{E B}
$$

\subsection{Discretization}

The variables of elastic deformation $u_{e}, v, w$, and $\phi$ depend on position and time. To descretize these variables they are separated into space-dependent shape functions $h(x)$ and time-dependent amplitudes $q(t)$. Thus

$$
\begin{aligned}
u_{e} & =h_{u}^{T}(x) q_{u}(t) & v & =h_{v}^{T}(x) q_{v}(t) \\
w & =h_{w}^{T}(x) q_{w}(t) & \phi & =h_{\phi}^{T}(x) q_{\phi}(t)
\end{aligned}
$$

Here $h$ and $q$ are vectors of length $N_{u}, N_{v}, N_{w}$ and $N_{\phi}$ where $N$ denotes the degree of freedom for each elastic variable. If $N=0$ for all degrees of freedom, the elastic beam degrades to a rigid body.

To keep the elastic motion separated from the rigid body motion, appropriate shape functions have to be chosen. Therefore the shape functions need to satisfy the boundary conditions $h(0)=0$ for elongation and torsion as well as $h(0)=h^{\prime}(0)=0$ for bending. This beam element employs algebraic polynomial shape functions as used in CAMRAD II; they are depicted in figure 3 for elongation and torsion as well as figure 4 for bending.

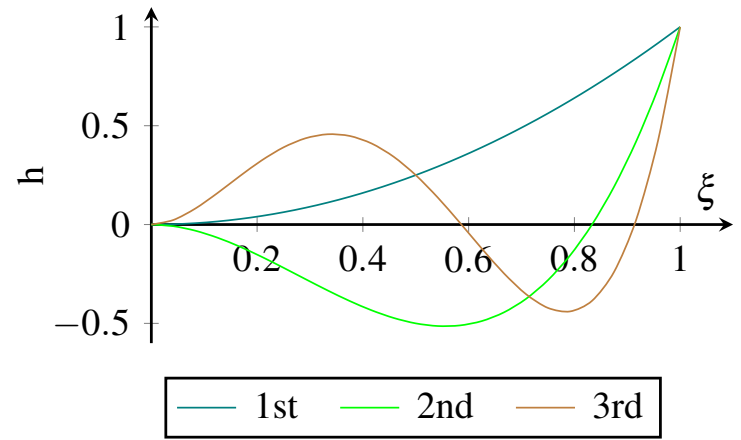

Figure 4: shape functions for $v$ and $w$

\subsection{Equations of Motion}

The equations of motion are derived from Hamilton's Principle:

$$
\delta \int \mathscr{L} \mathrm{d} t=\delta \int(T-U+W) \mathrm{d} t=0
$$

where $\mathscr{L}$ is the Lagrangian, $T$ the kinetic energy, $U$ the strain energy, and $W$ the work of external loads. Those terms will be explained in detail in the following sections. The strain energy is given by the product of stress $\sigma$ with the strain $\varepsilon$ integrated over the volume of the beam:

$$
\delta U=\int \delta \varepsilon^{T} \sigma \mathrm{d} \Omega
$$

Next, the work of the external loads can be expressed by integrating the body forces $b$, surface forces $t_{S}$ and discrete forces $F$ :

$$
\delta W=\int \delta p^{T} b \mathrm{~d} \Omega+\int \delta p^{T} t_{S} \mathrm{~d} \Gamma+\delta p^{T} F
$$

where $p$ is the position vector of a point on the beam relative to the inertial system (cf. fig. 2). Here surface forces $t_{S}$ will be discretized and can therefore be treated as discrete forces $F$. The only body force for the beam model is gravity, which will be treated as inertial force using d'Alembert's principle.

The kinetic energy can be obtained from the integral over density $\rho$ and the absolute beam velocity $\dot{p}$ :

$$
\delta T=\delta \int \frac{1}{2} \rho \dot{p}^{2} \mathrm{~d} \Omega
$$

Using partial integration in time with $\delta p=0$ at $t_{\text {initial }}$ and $t_{\text {final }}$ without loss of generality this can be expressed as (respecting gravitational forces $g$ as inertial force):

$$
\delta T=\int \delta p^{T}(-\ddot{p}+g) \rho \mathrm{d} \Omega
$$




\subsection{Kinetic Energy}

Equation (7) can be split into two integrals using the mass per length $m$ :

$$
-\delta T=\iint \delta p^{T}(\ddot{p}-g) \rho \mathrm{d} m \mathrm{~d} x
$$

with $p=s+C^{I B} r$, where $s$ is the vector from the inertial system to the beam origin and $r$ is the vector described in equation (1). The corresponding virtual displacement $\delta p$ can further be written as (with $\delta \psi$ as the virtual rotation of the beam origin $)^{2}$ :

$$
\begin{aligned}
\delta p= & \delta s-C^{I B} \widetilde{r} \delta \psi^{B I}+C^{I B} \delta r \\
= & C^{I B}\left[\begin{array}{llllll}
I & -\widetilde{r} & R_{u}^{T} & R_{v}^{T} & R_{w}^{T} & R_{\phi}^{T}
\end{array}\right] \\
& \left(\begin{array}{llllll}
\delta s^{*} & \delta \psi^{B I} & \delta q_{u} & \delta q_{v} & \delta q_{w} & \delta q_{\phi}
\end{array}\right)^{T} \\
= & C^{I B} R^{T} \delta q
\end{aligned}
$$

Here $R_{i}$ represents the Jacobian of the placement $r$ with respect to the degrees of freedom $u, v, w$, and $\phi$. Inserting (9) in (8) the kinetic energy becomes

$$
-\delta T=\delta q^{T} \iint R C^{I B}(\ddot{p}-g) \mathrm{d} m \mathrm{~d} x=\delta q^{T} M
$$

where $M$ is the resulting mass matrix. By differentiating $p$ twice the vector $\ddot{p}$ becomes

$$
\ddot{p}=C^{I B}(\ddot{s}+\widetilde{\omega} r+2 \widetilde{\omega} \dot{r}+\ddot{r}+\omega \dot{s}+\widetilde{\omega} \widetilde{\omega} r)
$$

Neglecting warping and transverse shear effects on inertia equation (1) can be written as:

$$
r=x^{E B}+C^{E B}\left(\begin{array}{c}
0 \\
\eta \\
\zeta
\end{array}\right)=x^{E B}+\left(Y_{-\beta} Z_{\zeta}\right)^{T}\left(\begin{array}{c}
0 \\
\eta_{b} \\
\zeta_{b}
\end{array}\right)
$$

Here $\eta_{b}$ and $\zeta_{b}$ identify the cross-section point, relative to the section principle axes at $\theta_{I}$ that are bent but not twisted. Thus the motion of a point on the crosssection is evaluated by

$$
r=\left(\begin{array}{c}
x+u \\
v \\
w
\end{array}\right)+\left(\begin{array}{c}
-S_{\zeta} \\
C_{\zeta} \\
0
\end{array}\right) \eta_{b}+\left(\begin{array}{c}
-S_{\beta} C_{\zeta} \\
-S_{\beta} S_{\zeta} \\
C_{\beta}
\end{array}\right) \zeta_{b}
$$

Consistent with the second order approximation and

$$
\left(\begin{array}{c}
\dot{\eta}_{b} \\
\dot{\zeta}_{b}
\end{array}\right)=\dot{\theta}\left(\begin{array}{c}
-\zeta_{b} \\
\eta_{b}
\end{array}\right) \approx \dot{\phi}\left(\begin{array}{c}
-\zeta_{b} \\
\eta_{b}
\end{array}\right)
$$

this can be reduced to (15) and derived twice:

$$
r=\left(\begin{array}{c}
x+u \\
v \\
w
\end{array}\right)+\left(\begin{array}{c}
-v^{\prime} \\
1 \\
0
\end{array}\right) \eta_{b}+\left(\begin{array}{c}
-w^{\prime} \\
0 \\
1
\end{array}\right) \zeta_{b}
$$

Now equation (15) can be derived twice and thus inserted in equation (11).

\footnotetext{
${ }^{2}$ In this paper the notation $(\widetilde{\cdot})$ will be used to denote the crossproduct matrix
}

\subsection{Strain Energy}

The strain energy $\delta U$ (cf. eq. (4)) is derived from the Green-Lagrange strain tensor, which is obtained by the basis vectors of the undistorted and distorted beam and can be written as

$$
f_{m n}=\frac{1}{2}\left(G_{m n}-g_{m n}\right)
$$

where $g_{m n}=\mathbf{g}_{m} \mathbf{g}_{n}$ and $G_{m n}=\mathbf{G}_{m} \mathbf{G}_{n}$ are the metric tensors in terms of the curvilinear coordinates $y_{m}=$ $(x, \eta, \zeta)$ of the undistorted and distorted beam, respectively. The basis vectors are defined as $\mathbf{g}_{m}=\partial r_{i} / \partial y_{m}$ and $\mathbf{G}_{m}=\partial r_{f} / \partial y_{m}$ with

$$
r_{i}=\left(\begin{array}{l}
x \\
0 \\
0
\end{array}\right)+X_{-\theta_{C}}\left(\begin{array}{l}
0 \\
\eta \\
\zeta
\end{array}\right)
$$

and $r_{f}=r$ as defined in equation (1). The GreenLagrange tensor $f_{m n}$ in curvilinear coordinates needs to be transformed into a stress tensor $\gamma_{k l}$ in local rectangular coordinates in order to apply the constitutive law. Thus the local Cartesian basis $z_{k}$ with the unit vectors $\mathbf{e}_{k}=\left(\mathbf{e}_{1}, \mathbf{g}_{2}, \mathbf{g}_{3}\right)$ is introduced. The transformation is then given by [10]:

$$
f_{m n}=\gamma_{k l} \frac{\partial z_{k}}{\partial y_{m}} \frac{\partial z_{l}}{\partial y_{n}}
$$

with

$$
\frac{\partial z_{k}}{\partial y_{m}}=\mathbf{e}_{k} \mathbf{g}_{m}=\left[\begin{array}{ccc}
1 & 0 & 0 \\
-\theta_{C}^{\prime} \zeta & 1 & 0 \\
\theta_{C}^{\prime} \eta & 0 & 1
\end{array}\right]
$$

The transformation results in the relations $\gamma_{11}=f_{11}+$ $2 \theta_{C}^{\prime}\left(\zeta f_{12}-\eta f_{13}\right), \gamma_{12}=f_{12}$ and $\gamma_{13}=f_{13}$. With the assumption of small strain, $\gamma_{m n}=\varepsilon_{m n}$, where $\varepsilon$ is linear in the strain measures. Then, after neglecting all bending and warping terms of higher order as well as warping in $y$-and $z$-direction the required strain $\varepsilon$ is:

$$
\begin{aligned}
\varepsilon_{11}= & \frac{1}{2}\left(G_{11}-g_{11}\right)+2 \theta_{C}^{\prime}\left(\zeta \varepsilon_{12}-\eta_{13}\right) \\
\approx & u_{e}^{\prime}-\kappa_{z} \eta+\kappa_{y} \zeta+1 / 2 \phi^{\prime 2}\left(\eta^{2}+\zeta^{2}\right) \\
& +2 \theta_{C}^{\prime}\left(\zeta \bar{\varepsilon}_{12}-\eta \bar{\varepsilon}_{13}\right)+\theta_{C}^{\prime} \phi^{\prime}\left(\zeta \lambda_{\eta}-\eta \lambda_{\zeta}\right) \\
2 \varepsilon_{12}= & G_{12}-g_{12} \approx 2 \bar{\varepsilon}_{12}+\left(\lambda_{\eta}-\zeta\right) \phi^{\prime} \\
2 \varepsilon_{13}= & G_{13}-g_{13} \approx 2 \bar{\varepsilon}_{13}+\left(\lambda_{\zeta}+\eta\right) \phi^{\prime}
\end{aligned}
$$

In this expression the warping function $W_{1}=\lambda \phi^{\prime}$ has been used.

In order to relate the strain $\varepsilon$ to the stress $\sigma$ used in equation (4) terms for section loads are needed. 
Assuming small strain, the sections loads can be expressed as linear combinations of the force strain measure $\gamma$ and the moment strain measure $\kappa$ (see ref. [6]):

$$
\begin{aligned}
& \gamma=C^{T}\left(\begin{array}{c}
1+u^{\prime} \\
v^{\prime} \\
w^{\prime}
\end{array}\right)-\left(\begin{array}{l}
1 \\
0 \\
0
\end{array}\right)=\left(\begin{array}{c}
\bar{\varepsilon}_{11} \\
2 \bar{\varepsilon}_{12} \\
2 \bar{\varepsilon}_{13}
\end{array}\right) \\
& \kappa=K-k
\end{aligned}
$$

with $\widetilde{K}=C^{T} C^{\prime}, \widetilde{k}=X_{\theta_{C}} X_{-\theta_{C}}^{\prime}$, and $k=\left(\begin{array}{lll}\theta_{C}^{\prime} & 0 & 0\end{array}\right)^{T}$. It can be shown that $K_{x}=\theta_{C}^{\prime}+\phi^{\prime}$, so $\kappa_{x}=\phi$ and $\gamma_{x}=$ $\bar{\varepsilon}_{11}=u_{e}^{\prime}$. Thus the second order approximation for $\gamma$ and $\kappa$ is:

$$
\begin{aligned}
& \gamma=X_{\theta}\left(\begin{array}{lll}
u_{e}^{\prime} & -v & -\omega
\end{array}\right)^{T} \\
& \kappa=X_{\theta}\left(\begin{array}{lll}
\phi^{\prime} & -w^{\prime \prime}-\omega^{\prime} & v^{\prime \prime}+v^{\prime}
\end{array}\right)^{T}
\end{aligned}
$$

For brevity the detailed derivation of the strain measures is omitted here, the reader is advised to refer to references [9] and [7]. The strain equations can now be inserted into the strain energy terms from Hamilton's principle. The stress is determined from strain by the constitutive law $\sigma_{i j}=E_{i j k l} \varepsilon_{k l}$, while only stresses acting perpendicular to the cross-section are taken into account. Thus only $\sigma_{11}, \sigma_{12}$ and $\sigma_{13}$ remain in the energy equations. Now equation (4) can be written in terms of section loads (forces $F_{i}$ and moments $M_{i}$ ):

$$
\begin{aligned}
& \delta U=\iint \delta \varepsilon^{T} \sigma \mathrm{d} A \mathrm{~d} x \\
&=\int_{0}^{l}\left[F_{x} \delta u_{e}^{\prime}+F_{y} 2 \delta \bar{\varepsilon}_{12}+F_{z} 2 \delta \bar{\varepsilon}_{13}\right. \\
&\left.\quad+M_{x} \delta \phi^{\prime}+M_{y} \delta \kappa_{y}+M_{z} \delta \kappa_{z}\right] \mathrm{d} x
\end{aligned}
$$

By integration of $\int \mathrm{d} A \delta \varepsilon E \varepsilon$ the section loads can be obtained from stress and hence related to the strain measures. In a next step the transverse shear forces are eliminated from the equations, the shear strain however will still be considered in the material parameters. That leads to the matrix of cross-sectional elastic constants $S$ :

$$
\begin{gathered}
\left(\begin{array}{c}
F_{x} \\
M_{x} \\
M_{y} \\
M_{z}
\end{array}\right)= \\
{\left[\begin{array}{cccc}
S_{u u} & S_{u \phi}+\frac{1}{2} \phi^{\prime} S_{u u} k_{P}^{2} & S_{u w} & S_{u v} \\
S_{\phi u}+\phi^{\prime} S_{u u} k_{P}^{2} & S_{\phi \phi} & S_{\phi w} & S_{\phi v} \\
S_{w u} & S_{w \phi} & S_{w w} & S_{w v} \\
S_{v u} & S_{v \phi} & S_{v w} & S_{v v}
\end{array}\right]\left(\begin{array}{c}
u_{e}^{\prime} \\
\phi^{\prime} \\
\kappa_{y} \\
\kappa_{z}
\end{array}\right)}
\end{gathered}
$$

These factors are required input data for the anisotropic beam model and can be obtained from the previously named beam analysis softwares in section 2. Here $k_{P}^{2}$ is the nonlinear coupling factor, which is the square of polar radius of gyration about the beam axis. For the isotropic model the matrix $S$ reduces to:

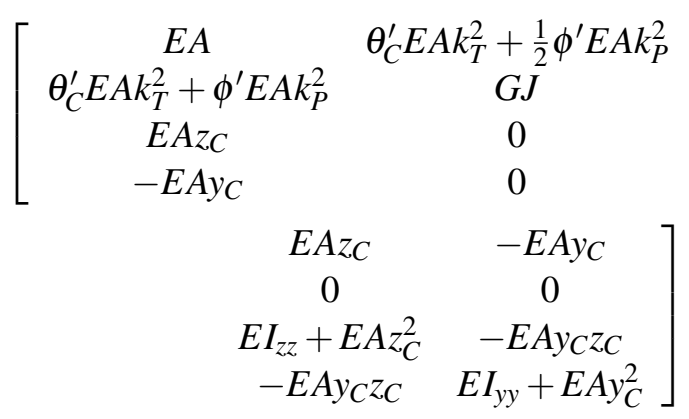

Where $y_{C}$ and $z_{C}$ is the horizontal and vertical offset of the tension center and $k_{T}^{2}$ the extension torsion coupling factor.

\section{Examples}

Calculations of the developed anisotropic flexible beam library have been compared to experimental measurements of the Princeton beam test as well as CAMRAD II simulation results. The static and dynamic behavior of the beam element has been evaluated for deflections, rotations and eigenfrequencies of the beam.

\subsection{Static Deflection - Princeton Beam Test}

The Princeton beam test [2] is an experimental study of the large static deformation of a cantilevered beam under gravity tip load. It involved an $20 \times 0.5 \times 0.125$ inch aluminum beam with a rectangular cross-section. The beam root is rotated around its principle axes so that the tip load is oriented at various angles. Static bending deflections of the tip have been measured as a function of tip load. The softer bending direction is called flap, the stiffer direction chord. The beam is fixed at the root in a way that at zero degrees rotation angle gravitational force deflects the beam chordwise. To compare the experimental results with the implemented beam model a different number of beam segments has been used. The cross-sectional data has been taken from reference [3]. Figure 5 shows the resulting bending deflection in parts of the beam length in flap direction. Figure 6 shows the corresponding results in chord direction.

It can be seen that significant nonlinear effects occur with increasing tip loads. Using one or two beam 


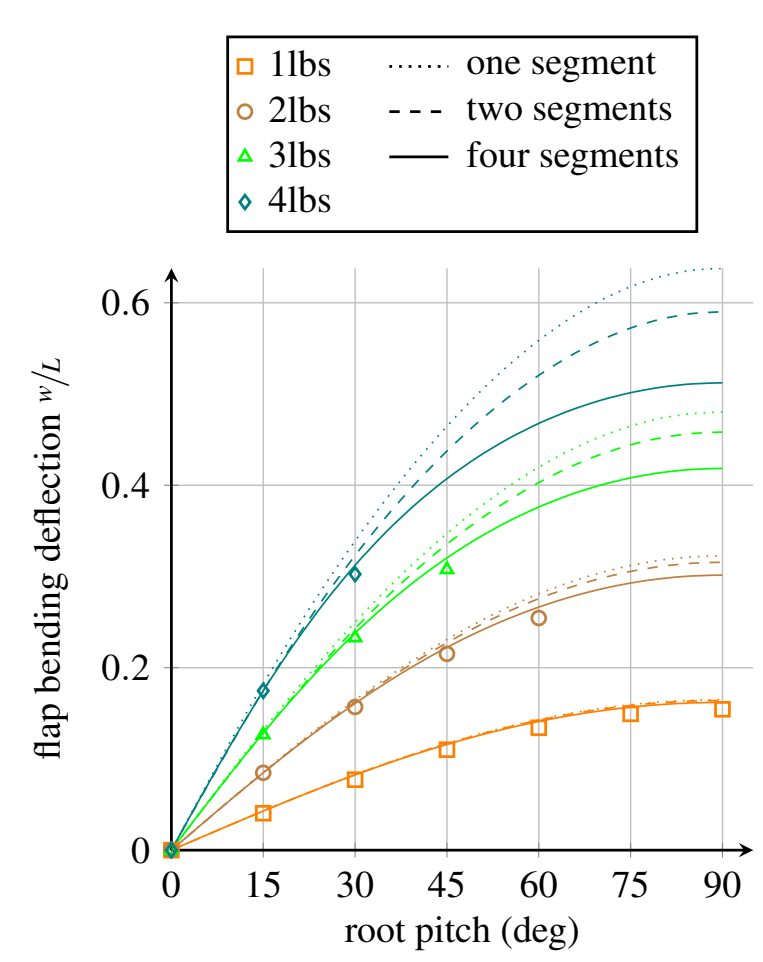

Figure 5: Princeton beam test: Comparison of flapwise tip bending deflection of measured and calculated (marks $=$ experimental data)

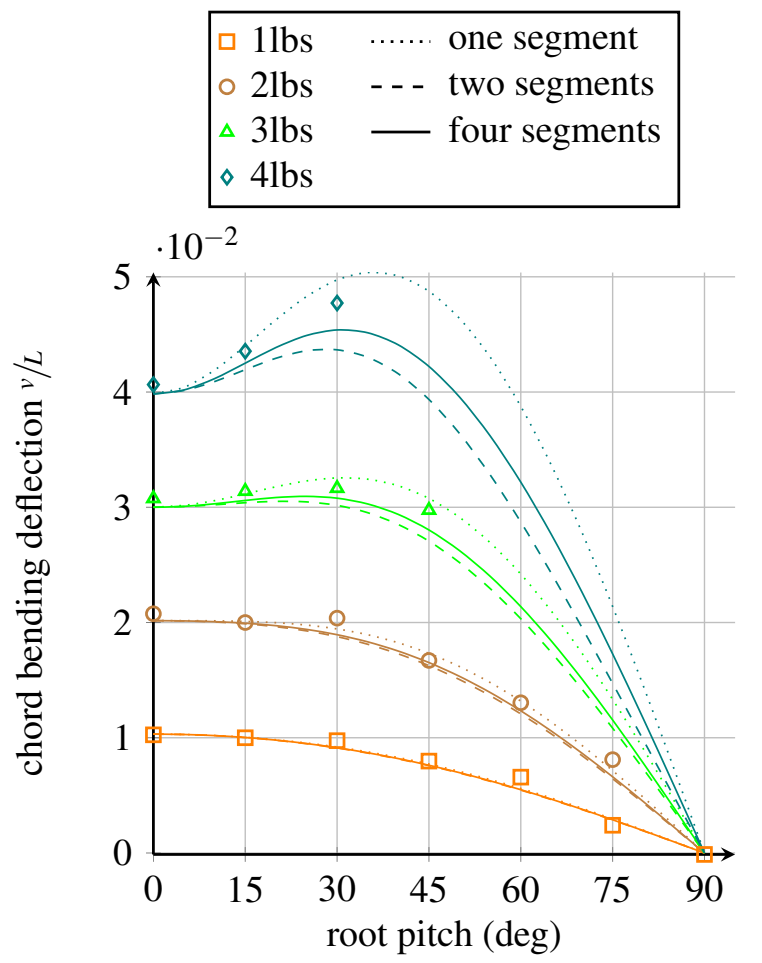

Figure 6: Princeton beam test: Comparison of chordwise tip bending deflection of measured and calculated $($ marks $=$ experimental data $)$

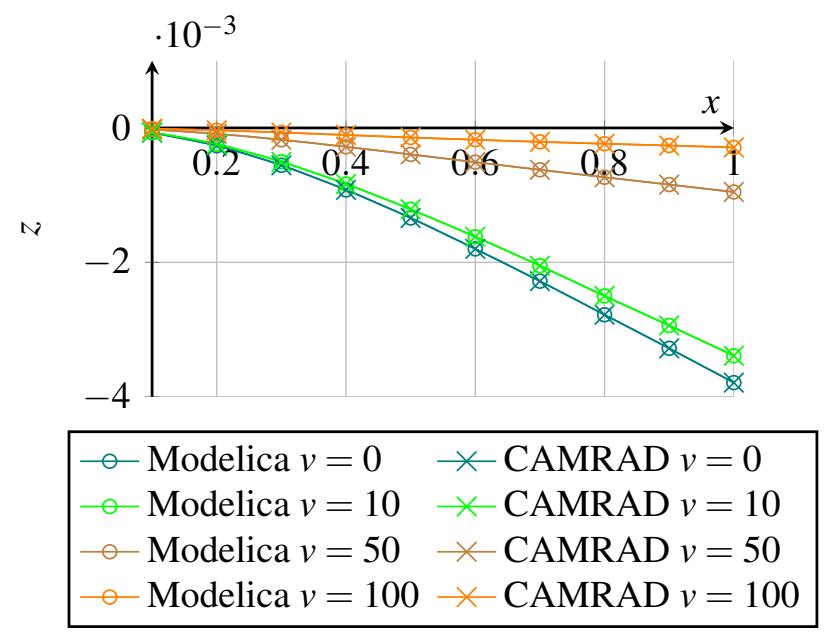

Figure 7: Vertical deflection of the Princeton beam at different tip speeds $v\left[\mathrm{~m} \mathrm{~s}^{-1}\right]$

segments the simulation is not capable of reproducing the experimental results due to the second order approximation. Using four beam segments however the large nonlinear deformation is captured by the rigid body motion, which is exact. In that case the simulation gives good results for flap and chord deflection.

\subsection{Dynamic Behavior}

To validate the dynamic behavior of the library a cantilever beam segment is rotated around its vertical axis at root using different tip speeds $v=\Omega r$, with $\Omega$ as the rotational speed. The transversal deflections in $x, y, z$-direction as well as the rotation angles $\theta,-\beta, \zeta$ around the $x, y, z$ axis respectively are measured with ten virtual sensors at equally spaced positions along the principal axis. To test the isotropic behavior crosssectional data from the Princeton beam test have been used. In order to analyze the anisotropic characteristics of the beam these parameters are expanded with structural and inertial coupling factors. This way more than 35 test beams have been created to vary all possible input parameters and compared the virtual Modelica measurements to CAMRAD II simulation results. Exemplary figure 7 depicts the vertical deflection of the isotropic Princeton beam. It can be shown, that the deviations between the two simulation softwares for all isotropic and anisotropic test cases are smaller than the predefined numerical tolerance of $e=10^{-5}$.

In rotorcraft analysis a widely used tool is a fan plot. They show the relation between the rotary speed and the eigenfrequency of a rotor. Again results are compared to CAMRAD II. The eigenfrequencies of the Modelica simulation are obtained using the "lin- 


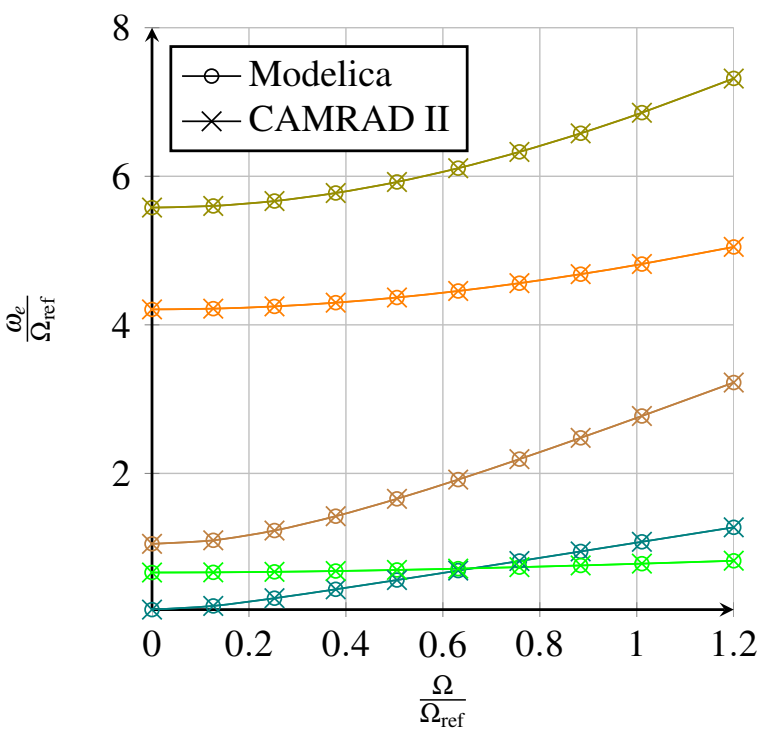

Figure 8: Fan plot of the first five eigenfrequencies for the Princeton beam. Abscissa: normalized eigenfrequency $\omega_{e}$; Ordinate: normalized rotary speed $\Omega$; reference speed $\Omega_{\mathrm{ref}}=380 \mathrm{rad} \mathrm{s}^{-1}$

earizeModel" function and extracting the eigenvalues with the Linear Systems Toolbox. Figure 8 shows the variation of the first five eigenfrequencies at different rotary speeds normalized with the reference speed of $\Omega_{\mathrm{ref}}=380 \mathrm{rad} \mathrm{s}^{-1}$. For all frequencies it can be shown that the results of both simulation software match perfectly.

\subsection{Animation}

The implemented anisotropic flexible beam library is capable of visualizing the deformations of the beam. For this purpose the Modelica surface visualizer from the MultiBody library is employed. Thus all standard features such as colors, transparency etc. are available. To make small deformations visible an amplification factor has been implemented which exaggerates all deformations and rotations of the beam segment in the animation window. To save computational power the resolution of the animation can be reduced or completely disabled. An example of the animation is presented in figure 9. Here four flexible beam elements are used to simulate a helicopter rotor blade (shown with exaggerated amplitudes), yet the consideration of aerodynamic forces is ongoing work.

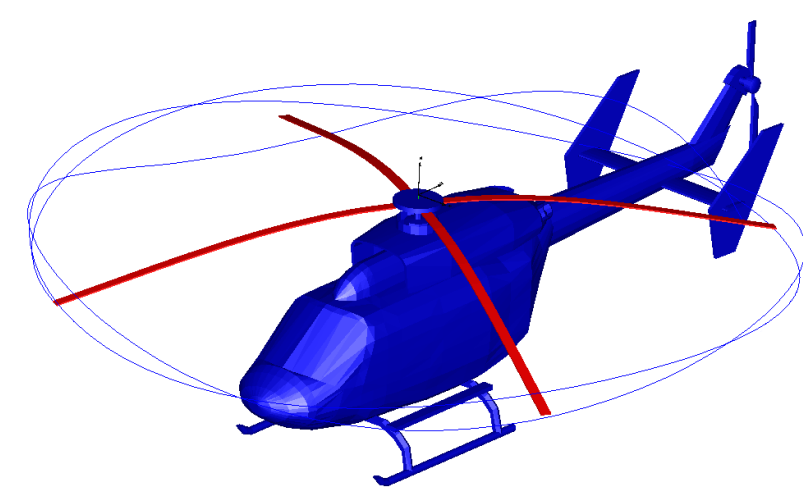

Figure 9: Animation of four elastic beam segments used as helicopter rotor blades

\section{Conclusions}

This paper presents a structural dynamic library to model anisotropic and nonhomogeneous elastic beams in Modelica. It is capable of simulating nonlinear extensional, torsional and flexural deformation and the couplings between those degrees of freedom. Using cross-sectional modeling theory the user is able to provide different varying material parameters along the beam principal axis. The results correlate with experimental beam measurements as well as other beam simulations software. To model large nonlinear deformation multiple beam segments can be connected in series.

\section{References}

[1] Cesnik, C. E. S. and D. H. Hodges: Variational-Asymptotical Analysis of Initially Curved and Twisted Composite Beams. Applied Mechanics Review, 46(11), 1993.

[2] Dowell, E. H., J. Traybar and D. H. HODGES: An experimental study of the nonlinear stiffness of a rotor blade undergoing flap, lag and twist deformations. NASA, 1975.

[3] Dowell, E. H., J. Traybar and D. H. HoDGES: An experimental-theoretical correlation study of non-linear bending and torsion deformations of a cantilever beam. Journal of Sound and Vibration, 50(4):533-544, 1977.

[4] Friedmann, P.P, B. Glaz and R. Palacios: A moderate deflection composite helicopter rotor blade model with an improved cross-sectional 
analysis. International Journal of Solids and Structures, 46(10):2186-2200, 2008.

[5] Giavotto, V. and M. Borri: Anisotropic Beam Theory and Applications. Computers and Structures, 16(1-4), 1983.

[6] Hodges, D. H.: Nonlinear composite beam theory. American Institute of Aeronautics and Astronautics, 2006.

[7] JOHnson, W.: CAMRAD II, Comprehensive Analytical Model of Rotorcraft Aerodynamics and Dynamics, 1992-1997.

[8] Johnson, W.: Rotorcraft dynamics models for a comprehensive analysis. 1998.

[9] SPIESS, C.: Entwicklung eines anisotropen Strukturdynamikmodells zur Simulation von elastischen Rotorblättern. Diploma Thesis, Technische Universität München, Garching, 2011.

[10] WASHIZU, K.: Variational methods in elasticity and plasticity. Pergamon press Oxford, UK, 1975. 